\section{P142 A COMPARISON OF THE SIMPLIFIED PULMONARY EMBOLISM SEVERITY INDEX WITH THE EUROPEAN SOCIETY OF CARDIOLOGY SEVERITY SCORE FOR ACUTE PE}

doi:10.1136/thoraxjnl-2012-202678.425

MT Harrison, JE Latham, JD Chalmers, S Schembri. NHS Tayside, Dundee, UK

Introduction Risk stratification in acute pulmonary embolism $(\mathrm{PE})$ is useful in identifying low risk patients suitable for ambulatory care and those at high risk in need of intensive monitoring.

In this study we compared two of the most well used scoring systems, the European Society of Cardiology (ESC) criteria and the simplified pulmonary embolism severity index (sPESI).

Methods We retrospectively identified patients admitted to NHS Tayside over a 4 year period with radiologically confirmed $\mathrm{PE}$ and calculated their ESC and sPESI scores.

RV dysfunction was defined by RV/LV ratio $>1$ on CTPA or by echocardiography. As per the ECS criteria, patients with shock (defined as $\mathrm{SBP}<90 \mathrm{mmHg}$ ) and RV dysfunction or myocardial injury (defined as troponin I $>0.045$ ) were classified as high risk, RV dysfunction or myocardial injury without shock as intermediate risk and patients with none of the above criteria classified as low risk.

Patients with any one of the six sPESI risk factors (age $>80$, cancer, chronic lung disease, $\mathrm{SpO}_{2}<90 \%, \mathrm{SBP}<90 \mathrm{mmHg}, \mathrm{HR}>110$ ) were classified as high risk.

The primary outcome was 30-day mortality or requirement for thrombolysis.

Results 291 patients (45.8\% male) were identified with a median age of 67 years (interquartile range $54-78$ years).

Low risk sPESI patients had a $2.2 \%$ risk of mortality or requirement for thrombolysis, in high risk patients (1 or more risk factors) the rate was $15.2 \%$. Using ESC criteria, low risk patients had a $7.0 \%$ risk or mortality or thrombolysis with high risk patients having a risk of $21.2 \%$.

The positive likelihood ratio for ESC was 2.2 vs 1.6 for sPESI. The negative likelihood ratio was 0.18 for sPESI compared to 0.7 for ESC.

The area under the receiver operator characteristic curves for both scores is shown in Figure 1. Both had moderate overall predictive value (AUC $0.68,95 \%$ CI $0.63-0.73$ ) for both scores, $p<0.0001$.

Conclusion The sPESI score is superior to the ESC score in identifying patients with acute $\mathrm{PE}$ who are at low risk of poor outcome, while ESC is superior to sPESI at identifying high risk patients.

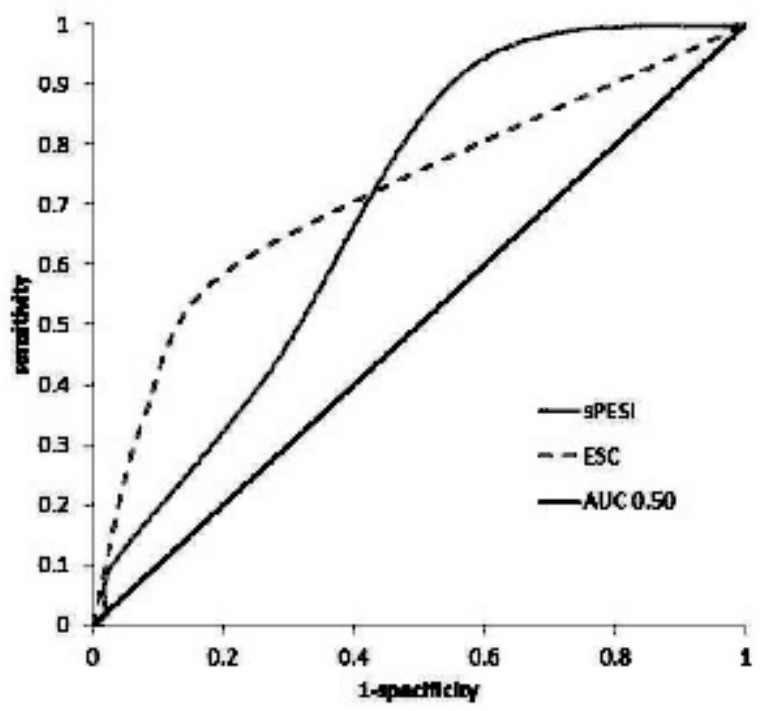

Abstract P142 Figure 1

\section{P143 A RETROSPECTIVE STUDY OF ECHOCARDIOGRAPHY AND FOLLOW-UP AFTER ACUTE PULMONARY EMBOLISM - ARE WE MISSING CHRONIC THROMBOEMBOLIC PULMONARY HYPERTENSION?}

doi:10.1136/thoraxjnl-2012-202678.426

D Slim, CN McBrien, LM O'Sullivan, SD Message, A Raghuram. Gloucestershire Hospitals NHS Foundation Trust, Gloucester, England

Introduction Pulmonary Hypertension (PH) is defined as raised Pulmonary Arterial Systolic Pressure $\geq 25 \mathrm{mmHg}$ at rest on Right Heart Catheterisation. ${ }^{1}$ Chronic Thromoboembolic Pulmonary Hypertension (CTEPH) is a recognised complication of pulmonary embolism (PE), with a reported annual cumulative incidence of $3.1 \%{ }^{2}$ Patients with evidence of PH or Right Ventricular Dysfunction (RVD) during admission should be referred for echocardiography usually 3-6 months after discharge to establish $\mathrm{PH}$ resolution. ${ }^{1}$ Objective To identify patients at risk of CTEPH, evaluate their follow-up plans and establish the proportion with evidence of acute $\mathrm{RVD} / \mathrm{PH}$ who are investigated for persistent $\mathrm{PH}$.

Methods Retrospective analysis of all diagnoses of PE on Computed Tomography Pulmonary Angiography (CTPA) in 2010 in a single Trust. Patients were stratified according to size and location of PE, and any reported radiological evidence of RVD. Echocardiography reports were reviewed for evidence of $\mathrm{PH}$.

Results $19.3 \%(329 / 1702)$ of CTPA scans revealed PE: Massive (28.6\%); Submassive (28.0\%); Peripheral (44.4\%). Only $17.6 \%(58 / 329)$ had inpatient echocardiography, with $55.1 \%(32 / 58)$ suggesting $\mathrm{PH}$ (PASP $\geq 36 \mathrm{mmHg}) .78 .1 \%(25 / 32)$ of these patients survived to 6 months and follow-up echocardiography was performed within 6 months for $40 \%(10 / 25)$ of this subset.

Overall, $80.9 \%(266 / 329)$ of patients with confirmed PE survived past 6 months. Follow-up echocardiogram was performed within 6 months on $20.3 \%$ (54/266) of survivors; $\mathrm{PH}$ was demonstrated in $18.5 \%(10 / 54)$.

RV strain was reported in $15.2 \%(50 / 329)$ of CTPA scans. Follow-up echocardiogram was performed within 6 months on $19.5 \%(8 / 41)$ of those alive at 6 months.

$84.2 \%(154 / 183)$ of individuals diagnosed with a massive or submassive PE survived to 6 months. Respiratory or cardiology followup was planned for $23 \%(36 / 154)$.

Conclusions Our findings suggest follow-up after acute PE is suboptimal, potentially missing early $\mathrm{PH}$ due to a low number of early echocardiograms. The relatively high percentage of $\mathrm{PH}$ on echocardiography compared to reported rates is likely due to selection bias. The results suggest there may be a missed cohort at risk of developing $\mathrm{PH}$, i.e. those with RV strain on CTPA or high thrombus load, being denied early or more aggressive interventions such as pulmonary endarterectomy.

\section{References}

1. Galié et al. EurRespirJ 2009; 34:1219-63.

2. Pengo et al. NEJM 2004; 350:2257-64.

\section{P144 CASE SERIES: CHOOSING THROMBOLYSIS IN ACUTE PULMONARY EMBOLISM (PE) - MASSIVENESS OR INSTABILITY?}

doi:10.1136/thoraxjnl-2012-202678.427

B Khan, R Lee, HA Raouf. Darent Valley Hospital, Dartford, Kent

Background PE has significant mortality risk particularly in "PE with haemodynamic instability" or "massive PE" where blood pressure, attributable to $\mathrm{PE}$, is sustained below $90 \mathrm{mmHg} \cdot{ }^{[1,2]}$ Thrombolysis in this patient group is associated with reduced mortality and faster restoration of lung perfusion with approval by NICE ${ }^{[1,2]}$ Another important group of patients with apparent haemodynamic stability but documented right ventricular (RV) compromise or 